\title{
Particle production vs. energy: how do simulation results match experimental measurements?
}

\section{M.BONESINI*}

Sezione INFN Milano Bicocca

E-mail: maurizio.bonesini@mib.infn.it

\begin{abstract}
This talk is about the available hadroproduction data to be used for the tuning of neutrino beamline simulations, going from conventional neutrino beams to superbeams and neutrino factories. Comparisons of data with existing Monte Carlo computations will be shown. Recent results from the HARP experiment at CERN PS will be summarized, including data useful for a better understanding of extended air showers and atmospheric neutrinos simulations.
\end{abstract}

10th International Workshop on Neutrino Factories, Super beams and Beta beams

June 30 - July 52008

Valencia, Spain

${ }^{*}$ Speaker. 


\section{Introduction}

The simulation of a conventional $v_{\mu}$ beam with a Monte Carlo (MC) is a delicate task due to complicate cascade processes involved in the neutrino production. The paucity of available hadroproduction data, needed for MC tuning, can limit systematically the precision in the calculations. In addition, new hadroproduction data at low energies are of great interest for extended air showers (EAS) and atmospheric neutrinos simulations and for the neutrino factory (NF) design. One relevant point is how existing MC simulations compare to available hadroproduction data. The more recent dedicated hadroproduction experiments in the field are NA56/SPY [1], MIPP [2], NA61 [3] and HARP [4]. At low energies $(\leq 15 \mathrm{GeV})$, the main experimental results come from the HARP experiment at CERN PS and will be briefly summarized here. At higher energies we refer to [5] for further details.

\section{The HARP experiment at CERN PS}

The HARP experiment at CERN PS [4] was designed to study hadroproduction on nuclear targets (from $\mathrm{H}_{2}$ to Ta) in the incident momentum range between 3 and $15 \mathrm{GeV} / \mathrm{c}$. The HARP detector is shown in Fig. 1 and includes different subdetectors for tracking and particle identification (PID) over the full solid angle. At large angle $\left(20^{\circ} \leq \theta \leq 160^{\circ}\right)$ tracking and PID are performed by a TPC and an array of RPC counters. In the forward direction $\left(\theta \leq 14.3^{0}\right)$ the tracking device is a set of drift chambers, recovered from the previous NOMAD experiment, while the PID is provided by a threshold Cerenkov counter, a time of flight wall (TOFW) and an e.m. calorimeter [6]. Beam particles are tagged by a system of beam TOF detectors (TOFA,TOFB) and Cherenkov counters. Data were taken in 2001 and 2002, for a total of about 420 M triggers in $\sim 300$ experimental settings.

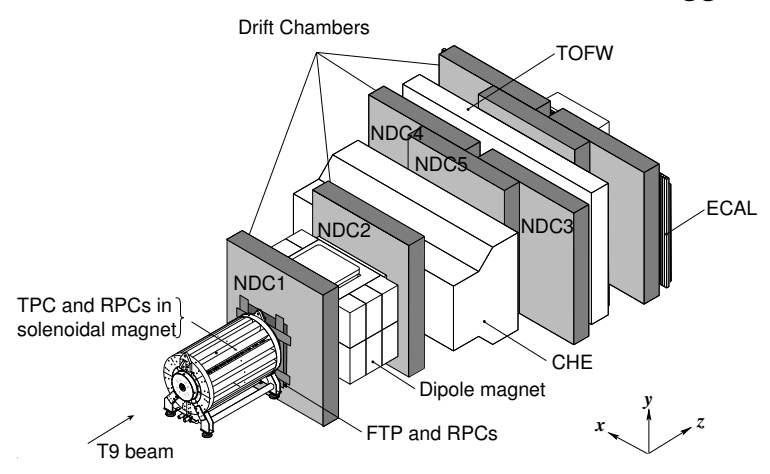

Figure 1: Layout of the HARP experiment at CERN PS.

\section{Results for simulation of NF beams.}

The baseline option for a NF target is a $\mathrm{Hg}$ jet target with impinging particles at energies $10 \pm 5 \mathrm{GeV}$. Available data are very scarce and for the MC tuning the HARP data on heavy targets, such as $\mathrm{Ta}$ or $\mathrm{Pb}$, are of utmost importance. The kinematical coverage of the HARP experiment is compared with the acceptance of a typical NF design in figure 2. The experiment covers the full momentum range of interest for production angles bigger than $0.35 \mathrm{rad}$. The pion yield increases 

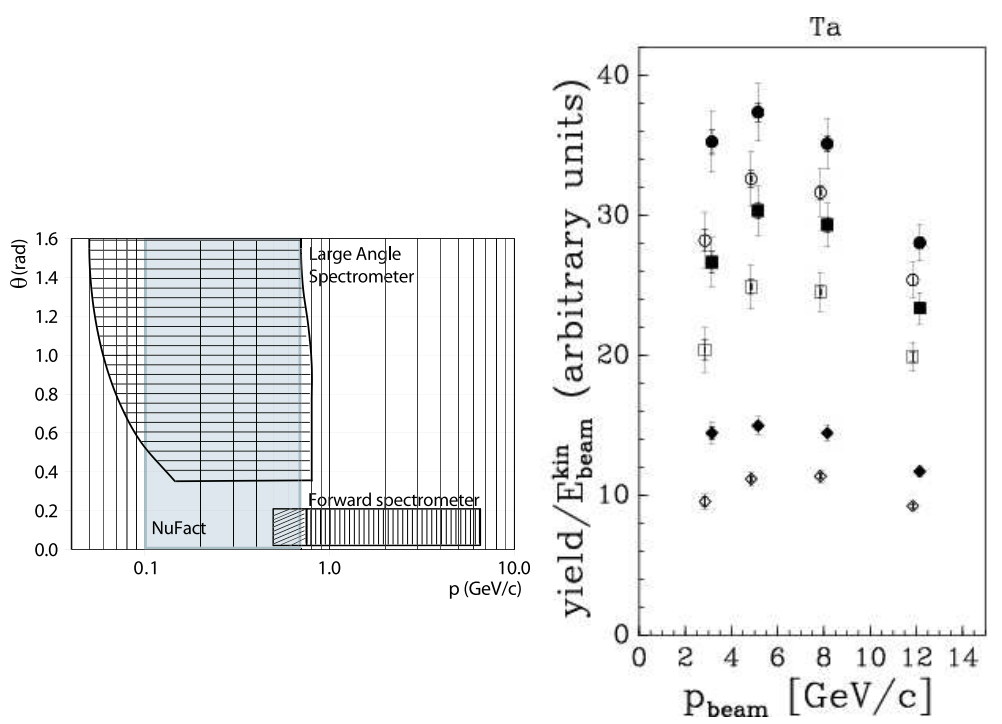

Figure 2: Left panel: kinematic region in the $p-\theta$ plane covered by HARP as compared to the acceptance of the input stage of typical NF designs. Right panel: $\pi^{+}$(closed symbols) and $\pi^{-}$(open symbols) yields as a function of the incident proton beam momentum for different design of the NF focussing stage. The circles indicate the integral over the full HARP acceptance, the squares are integrated over $0.35 \mathrm{rad} \leq \theta \leq$ $0.95 \mathrm{rad}$, while the diamonds require in addition the momentum cut $250 \mathrm{MeV} / \mathrm{c} \leq p \leq 500 \mathrm{MeV} / \mathrm{c}$.

linearly with momentum and has an optimum between $5 \mathrm{GeV} / \mathrm{c}$ and $8 \mathrm{GeV} / \mathrm{c}$, as can be seen in the right panel of figure 3. Final results for pion production on heavy targets have been published in reference [7] and some comparisons with available MC simulations are outlined in figure 3. None of the considered models describe fully HARP data. However, $\pi^{+}$production is described better than $\pi^{-}$production. At lower (higher) energies binary and Bertini models from GEANT4 (the FTP model from GEANT4 and MARS) seem more appropriate. Parametrized models (such as LHEP from GEANT4) show relevant discrepancies, up to a factor 3.

\section{Results for simulation of EAS and atmospheric neutrinos}

Results on cryogenic targets, such as $N_{2}$ and $O_{2}$ have a direct impact on the precise calculation of atmospheric neutrino fluxes and on the improved reliability of extensive air shower simulations by reducing the uncertainties of hadronic interaction models in the low energy range. In particular, the common hypothesis that $\mathrm{p}-\mathrm{C}$ data can be used to predict the $\mathrm{p}-\mathrm{N}_{2}$ and $\mathrm{p}-\mathrm{O}_{2}$ pion production cross-sections may be tested. HARP has published results [8] on charged pion production cross-sections in interactions of $12 \mathrm{GeV} / \mathrm{c}$ protons on $\mathrm{C}, \mathrm{O}_{2}$ and $\mathrm{N}_{2}$ thin targets, in the kinematic range $0.5 \mathrm{GeV} / \mathrm{c} \leq p_{\pi}<8 \mathrm{GeV} / \mathrm{c}$ and $50 \mathrm{mrad} \leq \theta_{\pi}<250 \mathrm{mrad}$. Some results, showing also a comparison with available simulations, are reported in figure 4.

\section{Results for simulation of conventional neutrino beams: the MiniBooNE and K2K physics cases.}

Prediction of the far detector spectrum in the absence of oscillations is a key ingredient in a 


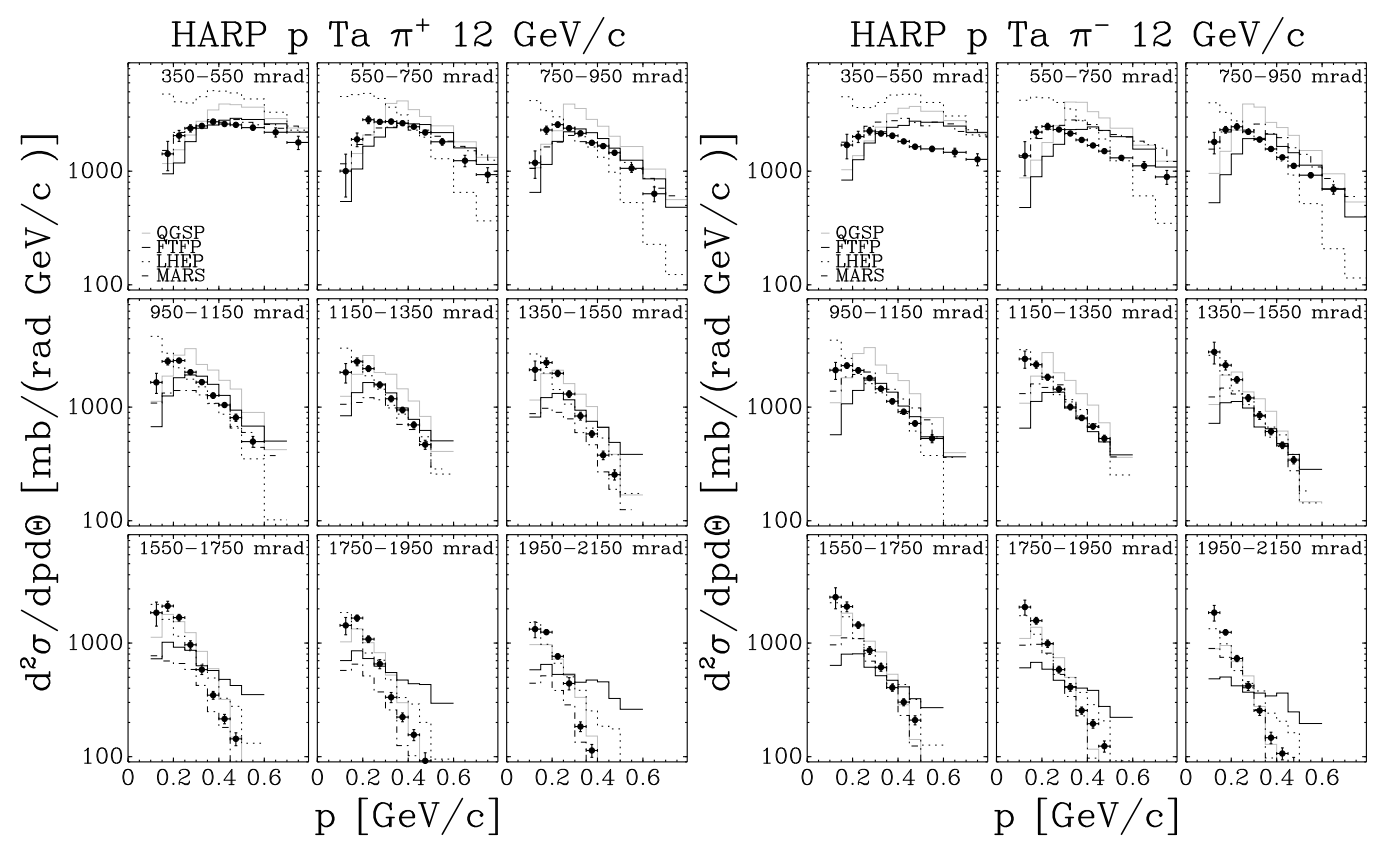

Figure 3: Experimental results from HARP at $12 \mathrm{GeV} / \mathrm{c}$ for $\mathrm{p}$-Ta cross sections for $\pi^{ \pm}$production, as compared to MC models. See [7] for further details and more data-MC comparisons.
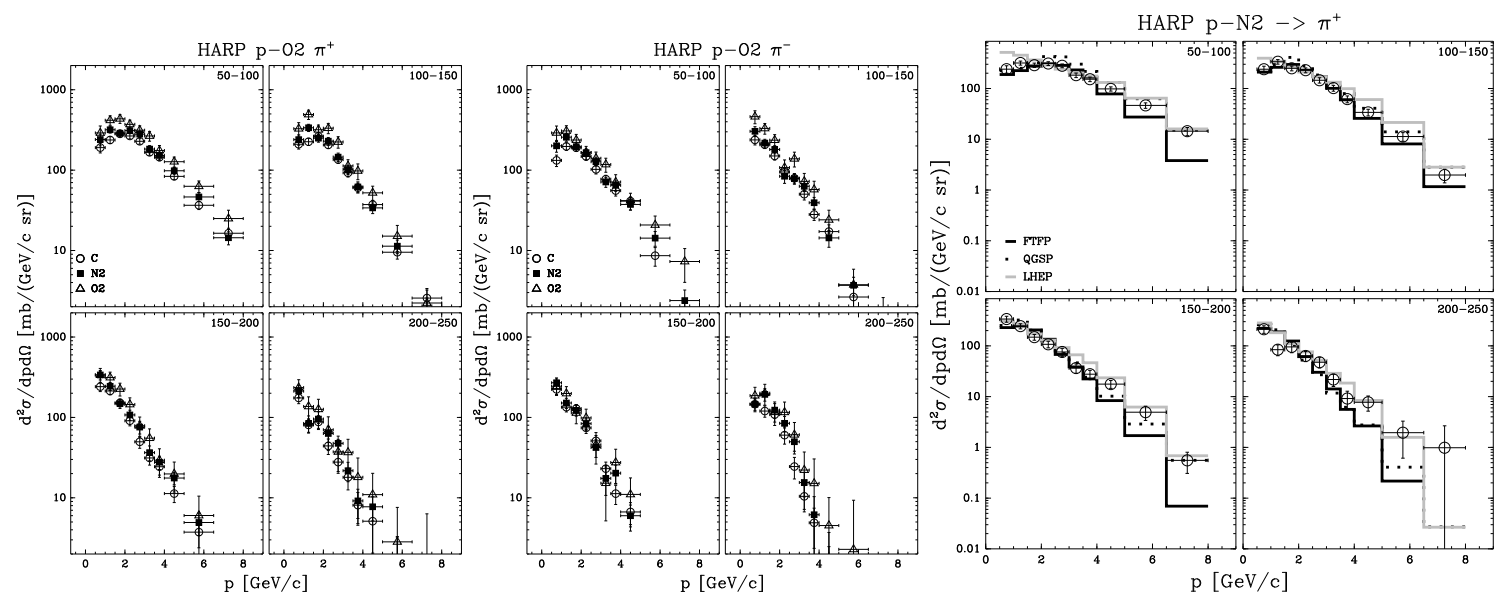

Figure 4: Left: $\mathrm{p}-\mathrm{O}_{2}, \mathrm{p}-\mathrm{N}_{2}, \mathrm{p}-\mathrm{C}$ cross sections at $12 \mathrm{GeV} / \mathrm{c}$; right: comparison of $\pi^{+}$production in $\mathrm{p}-\mathrm{N}_{2}$ interactions with different MC models from GEANT4.

neutrino oscillation experiment. This can be done by an extrapolation from a near detector via a nominal far/near ratio estimated by a beamline MC simulation. The error on the observed number of events in the $\mathrm{K} 2 \mathrm{~K}$ far detector (SuperKamiokande) was dominated by contributions from uncertainties of normalization $( \pm 5 \%)$ and far/near ratio $( \pm 5 \%)$.

HARP has reported measurements of the $\pi^{+}$production in $\mathrm{p}-\mathrm{Al}$ interactions at $12.9 \mathrm{GeV} / \mathrm{c}$ [9]. These results have contributed in a significant way to reduce the systematic error associated to the FAR/NEAR ratio, thus increasing the K2K sensitivity to oscillation signals [10]. 
Similar results were obtained in $8.9 \mathrm{GeV} / \mathrm{c}$ p-Be interactions [11] and have contributed to a better understanding of the MiniBooNE and SciBooNE $v$ fluxes. Figure 5 reports the comparison with some available MC models.

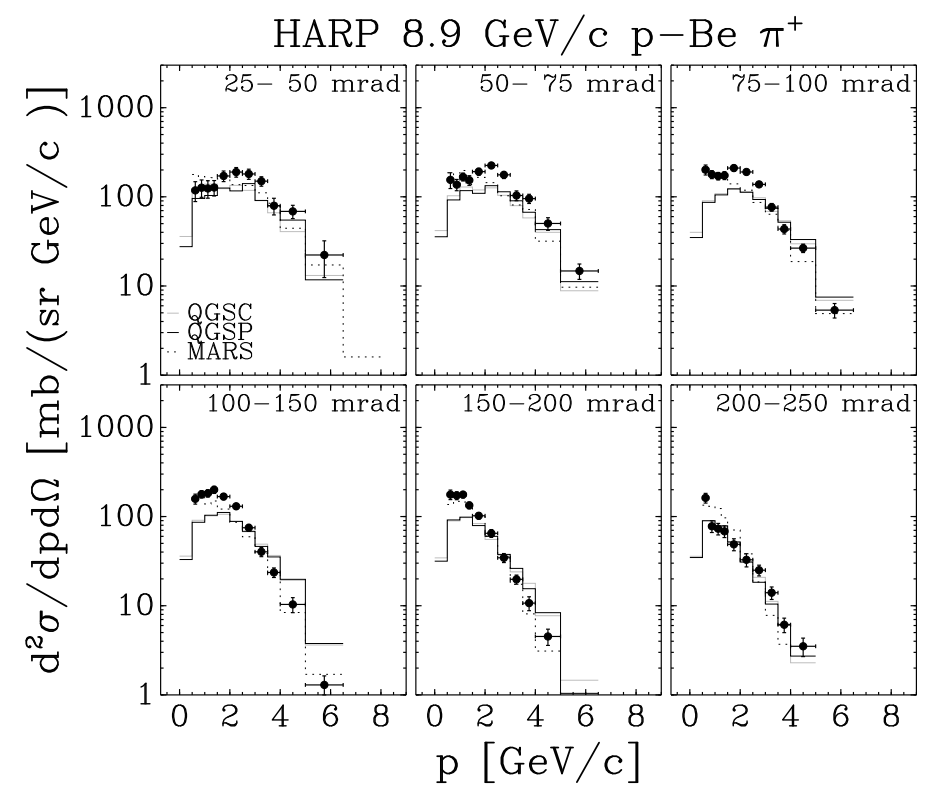

Figure 5: Experimental results from HARP at $8.9 \mathrm{GeV} / \mathrm{c}$ for $\mathrm{p}$-Be cross sections for $\pi^{+}$production, as compared to MC models from GEANT4 (QGSC,QGSP) and MARS.

\section{References}

[1] G. Ambrosini et al., NA56/SPY Collaboration, Eur. J. Phys. C10 (1999) 605 ;

M. Bonesini et al. Eur. J. Phys. C20 (2001) 13.

[2] MIPP Collaboration, http://ppd.fnal.gov/experiments/2907.

[3] N. Antoniou et al., NA61 Collaboration, CERN-SPSC-P-330, 2006.

[4] M.G. Catanesi et al., CERN-SPSC/99-35, SPSC/P315, 15 November 1999.

[5] M. Bonesini, A. Guglielmi, Phys. Rep. 433 (2006) 65.

[6] M. G. Catanesi et al., Nucl. Instr. Meth. A571 (2007) 527;

M. Baldo-Ceolin et al., Nucl. Instr. Meth. A532 (2004) 548.

[7] M.G. Catanesi et al., HARP Collaboration, Phys. ReV. C77 (2008) 0555207

M.G. Catanesi et al., HARP Collaboration, Eur. Phys. J. C51 (2007) 787.

[8] M.G. Catanesi et al., HARP Collaboration, Astr. Phys. 29 (2008) 257;

M.G. Catanesi et al., HARP Collaboration, "Forward $\pi^{ \pm}$production in $\mathrm{p}-\mathrm{O}_{2}$ and $\mathrm{p}-\mathrm{N}_{2}$ interactions at $12 \mathrm{GeV} / \mathrm{c} "$, in press on Astr. Phys.

[9] M.G. Catanesi et al., HARP Collaboration, Nucl. Phys. B732 (2006) 1.

[10] M.H. Ahn et al., K2K Collaboration, Phys. ReV.D74 (2006) 072003.

[11] M.G. Catanesi et al., HARP Collaboration, Eur. Phys. J. C52 (2007) 49. 